\section{Fatores de risco e de proteção para câncer de mama: uma revisão sistemática}

\author{
Risk and protective factors for breast cancer: \\ a systematic review
}

\author{
${ }^{1}$ Faculdade de Nutrição, \\ Universidade Federal de \\ Goiás, Goiânia, Brasil. \\ Correspondência \\ M. M. V. Naves \\ Faculdade de Nutrição, \\ Universidade Federal de Goiás. \\ Rua 227, Qd. 68, s/n, Setor \\ Leste Universitário, Goiânia \\ GO 74605-080, Brasil. \\ mnaves@fanut.ufg.br
}

\section{Abstract}

This review aimed to investigate risk and protective factors for breast cancer and to analyze whether scientific evidence from the World Cancer Research Fund and American Institute for Cancer Research, published in 2007, was confirmed by new research. In May 2010 we reviewed cohort and case-control analytical studies from 2007 to 2010 in the PubMed, LILACS, and SciELO databases. We selected 27 articles (14 case-control and $13 \mathrm{co}$ hort studies). Breastfeeding and physical activity were protective factors against breast cancer, and alcohol consumption was a risk factor. A direct proportional relationship was observed between larger waist circumference, weight throughout adulthood, and height and risk of breast cancer in postmenopausal women. The association between body fat and breast cancer is contradictory in both premenopausal and postmenopausal women. According to the accumulated evidence, breastfeeding and healthy lifestyle are the factors most strongly associated with breast cancer prevention.

Breast Neoplasms; Anthropometry; AlcoholDrinking; Motor Activity; Lactation
Livia Emi Inumaru 1

Érika Aparecida da Silveira 1

Maria Margareth Veloso Naves ${ }^{1}$

\section{Introdução}

O câncer consiste em uma enfermidade crônica, caracterizada pelo crescimento celular desordenado, o qual é resultante de alterações no código genético. Entre 5\% a 10\% das neoplasias são resultados diretos da herança de genes relacionados ao câncer, mas grande parte envolve danos ao material genético, de origem física, química ou biológica, que se acumulam ao longo da vida 1,2 .

O câncer de mama é o segundo tipo de câncer mais frequente no mundo e o mais comum entre as mulheres, e sua incidência vem aumentando ao longo do tempo, concomitantemente ao aumento da industrialização e da urbanização 1,3 . A neoplasia maligna de mama é responsável por cerca de $20 \%$ da incidência de câncer e por $14 \%$ do total de mortes associadas às neoplasias, entre as mulheres 1 .

O World Cancer Research Fund (WCRF) e o American Institute for Cancer Research (AICR) realizaram ampla investigação sobre os fatores associados ao câncer de mama, conforme as evidências científicas disponíveis até 2007 1. Variáveis ginecológicas, antropométricas, história da amamentação, ingestão de bebida alcoólica e atividade física mereceram destaque neste documento. Porém, ainda existem muitos aspectos a esclarecer, principalmente com relação à composição corporal e à atividade física 1 , tornando necessária a realização de mais estudos sobre a 
influência destes fatores na proteção ou risco para a neoplasia maligna de mama.

As evidências de que o câncer pode ser prevenido vêm de estudos internacionais em que se observaram variações nas taxas de incidência de câncer segundo as variáveis tempo e lugar. Populações que migram do país de origem para outras localidades apresentam mudanças nas taxas de incidência de câncer, o que demonstra que as neoplasias são, em parte, influenciadas pelas condições ambientais, não sendo determinadas apenas por fatores genéticos 4 . Segundo o Instituto Nacional de Câncer (INCA) 3, pelo menos um terço de casos novos de câncer que ocorrem no mundo anualmente poderiam ser prevenidos.

Apesar da importância de se conhecer os fatores envolvidos na etiologia do câncer de mama, visto que é um importante problema de Saúde Pública mundial, alguns deles ainda não estão bem elucidados. Diante do exposto, o objetivo desta revisão sistemática foi investigar alguns fatores de risco e de proteção para o câncer de mama: composição corporal, consumo de bebida alcoólica, atividade física e amamentação. Outro objetivo foi avaliar se as relações estabelecidas pelo WCRF e AICR 1 sofreram alterações em relação às novas evidências científicas publicadas recentemente.

\section{Metodologia}

Realizou-se uma revisão sistemática da literatura referente aos estudos de delineamento coorte e caso-controle, com enfoque populacional e hospitalar, publicados nos últimos quatro anos (2007 a 2010), nas bases PubMed (http://www. pubmed.gov), LILACS (http://bases.bireme.br) e SciELO (http://www.scielo.org). Foram delimitados os últimos quatro anos, uma vez que o trabalho do WCRF e AICR 1 incluiu estudos publicados até o ano de 2006.

A busca foi realizada em maio de 2010, e os limites usados para a pesquisa bibliográfica na base PubMed foram: materiais referentes aos últimos cinco anos, em língua inglesa ou espanhola, modelos humanos e gênero feminino. Os descritores usados para a obtenção de estudos caso-controle foram: "breast cancer", "risk factors"e "case-control". Desta forma, foram recuperados 1.055 artigos, dos quais somente 13 foram selecionados, conforme os critérios de inclusão e exclusão mostrados na Tabela 1. Já para a obtenção de estudos coorte, foram usados os seguintes descritores: "breast cancer", "risk factors" e "cohort”, recuperando-se, assim, 1.020 estudos. Destes, somente 14 foram selecionados, segundo os critérios pré-definidos.

Em relação à base de dados LILACS, a busca foi feita por meio do formulário básico, usandose os descritores "câncer de mama e fatores de risco e caso-controle", e "câncer de mama e fatores de risco e coorte", recuperando-se 18 e 3 artigos, respectivamente. Destes, nenhum atendeu aos critérios de inclusão do presente estudo.

Já a busca na base SciELO foi realizada por meio do método integrado e referente à área de Saúde Pública. Os descritores usados foram: "breast cancer, risk factors, case control" e "breast cancer, risk factors, cohort”, recuperando-se, assim, 8 e 3 artigos, respectivamente, dos quais nenhum

Tabela 1

Critérios de inclusão e exclusão de artigos para revisão sistemática.

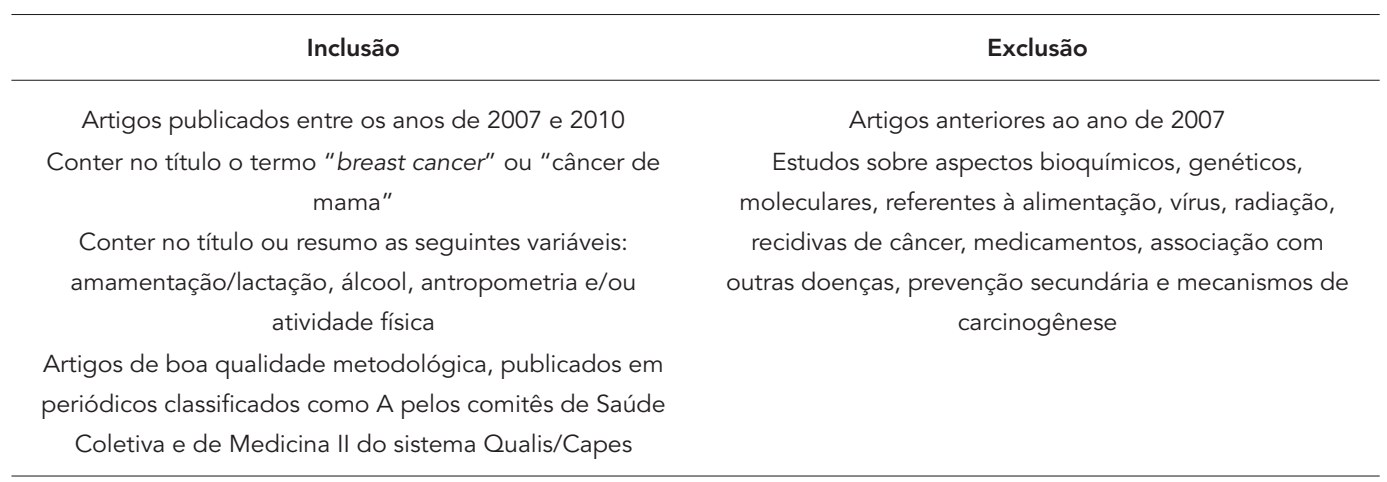

Capes: Coordenação de Aperfeiçoamento de Pessoal de Nível Superior. 
foi selecionado para o presente estudo por não atender aos critérios estabelecidos (Tabela 1).

Desta forma, foram objetos desta revisão 27 artigos, dos quais 13 são do tipo caso-controle e 14, do tipo coorte. Além destes, ao longo desta revisão foram citados outros documentos para fundamentação teórica e discussão do tema.

As evidências discutidas nesta revisão foram classificadas como convincentes, prováveis ou inconclusivas, conforme os seguintes critérios adotados pelo WCRF e o AICR 1: convincentes, quando confirmadas por pelo menos dois estudos coorte e por evidência experimental; prováveis, quando confirmadas por pelo menos dois estudos coorte ou cinco estudos do tipo casocontrole; e inconclusivas, quando o corpo de evidências era limitado em número de estudos disponíveis e, ou, em qualidade dos estudos. Nas classificações convincentes e prováveis, adotadas pelo WCRF e o AICR 1, os estudos deveriam ser independentes (estudos desenvolvidos por grupos de pesquisa independentes), possuir evidências de plausibilidade biológica e boa qualidade científica (estudos bem delineados, ausentes de erros metodológicos, tais como de seleção e de dimensionamento da amostra, de medição e de análise estatística).

\section{Resultados}

Fatores de proteção para o câncer de mama

\section{- Lactação}

A prática da lactação é apontada pelo WCRF e AICR ${ }^{1}$ como fator protetor convincente para a neoplasia maligna de mama, tanto em mulheres na pré-menopausa quanto na pós-menopausa (Tabela 2). Porém, ainda não há consenso sobre o tempo de amamentação que exerce esta proteção contra o câncer de mama.

Estudos recentes 5,6,7,8,9 confirmam os achados do WCRF e AICR 1 . Huo et al. 5, em um estudo caso-controle desenvolvido na Nigéria com uma amostra de 819 casos e 569 controles, encontraram uma redução de $7 \%$ no risco de desenvolver este tipo de câncer a cada aumento de 12 meses no tempo de amamentação [odds ratio $(\mathrm{OR})=$ 0,93; intervalo de 95\% de confiança (IC95\%): 0,87$1,0]$. Além disso, neste mesmo estudo, verificouse um efeito protetor do tempo de amamentação total para a neoplasia maligna de mama, quando o período de lactação foi superior a 49 meses, comparando-se com mulheres que amamentaram por 24 meses ou menos ( $p=0,005)$.

Beaber et al. 6 estudaram a relação entre a prática da amamentação e o câncer de mama, segundo o tipo da doença (ductal, lobular ou ductal-lobular) e verificaram efeito protetor significativo apenas para a neoplasia ductal, sugerindo que os diferentes tipos de câncer de mama podem ter diferentes etiologias. Observaram um OR igual a 0,7 (IC95\%: 0,5-0,9) para mulheres que amamentaram, e o mesmo valor de OR para a categoria de amamentação total entre 1 mês e 5,9 meses, tendo como referência, em ambos os casos, as mulheres que nunca amamentaram.

Stuebe et al. 7, em uma coorte de $60.075 \mathrm{mu}-$ lheres dos Estados Unidos, encontraram um risco relativo (RR) de 0,75 (IC95\%: 0,56-1,0) para as mulheres que amamentaram, comparando-se àquelas que nunca amamentaram. Ao analisar o tempo de amamentação, não se observou associação significativa. Resultado semelhante foi encontrado por Bessaoud \& Daurès 8 , em um estudo realizado na França com 437 casos e 922 controles, cujo valor de OR foi igual a 0,71 (IC95\%: 0,56-0,89) para as mesmas categorias (amamentação presente ou ausente). Interessante observar também que no estudo de Stuebe et al. 7 foram realizadas as análises do período de aleitamento materno exclusivo e do período de lactação com amenorréia, e não foram encontradas associações significativas.

Gajalakshmi et al. 9, em um estudo desenvolvido na Índia com 1.866 casos e 1.873 controles, fizeram a análise dos dados segundo o status menopausal, e observaram efeito protetor da amamentação somente para mulheres na pré-menopausa, a partir do tempo de dois anos de lactação total, tomando-se como referência o tempo de amamentação igual ou inferior a um ano.

Lord et al. 10 investigaram a associação entre as variáveis “idade na primeira gestação", "paridade", "amamentação" e "risco de câncer de mama", e constataram que a lactação pode exercer um papel protetor independente de outros fatores. Fez-se a análise das mulheres que tiveram filho após os 25 anos de idade e verificou-se que aquelas que amamentaram apresentaram um valor de OR igual a 0,57 (IC95\%: 0,37-0,87), comparandose àquelas que nunca amamentaram. Este resultado indica que a lactação pode inibir os efeitos negativos da gestação tardia, conforme já relatado pelo Collaborative Group on Hormonal Factors in Breast Cancer, um grupo de pesquisa que avalia os fatores hormonais e sua relação com a neoplasia maligna de mama 11.

Apesar do efeito protetor da lactação ter sido confirmado por grande parte dos estudos, Nemesure et al. 12 não encontraram associação significativa em estudo caso-controle realizado em Barbados com uma amostra de 241 casos e 481 controles. 
Tabela 2

Fatores de risco e de proteção para o câncer de mama, segundo estudos publicados entre 2007 e 2010, em comparação com os achados do WCRF e AICR 1.

\begin{tabular}{|c|c|c|c|c|}
\hline \multirow[t]{2}{*}{ Variável } & \multirow[t]{2}{*}{ WCRF/AICR } & \multicolumn{3}{|c|}{ Resultados dos artigos publicados entre 2007 e 2010} \\
\hline & & Fator de risco & Fator de proteção & $\begin{array}{c}\text { Associação não } \\
\text { significativa }\end{array}$ \\
\hline Lactação & $\begin{array}{l}\text { Fator convincente de } \\
\text { proteção tanto na pré- } \\
\text { menopausa quanto na } \\
\text { pós-menopausa }\end{array}$ & - & $\begin{array}{c}\text { Huo et al. (2008) 5, } \\
\text { Beaber et al. (2008) 6, } \\
\text { Stuebe et al. (2009) 7, } \\
\text { Bessaoud \& Daurès (2008) 8, } \\
\text { Gajalakshmi et al. (2009) } 9\end{array}$ & Nemesure et al. (2009) 12 \\
\hline Atividade física & $\begin{array}{c}\text { Pré-menopausa: } \\
\text { fator limitado/sugestivo } \\
\text { de proteção }\end{array}$ & - & $\begin{array}{l}\text { Sprague et al. (2007) } 13 *, \\
\text { Lahmann et al. (2007) } 14, \\
\text { Suzuki et al. (2008) 19, } \\
\text { Peplonska et al. (2008) } 16\end{array}$ & - \\
\hline & $\begin{array}{l}\text { Pós-menopausa: fator } \\
\text { provável de proteção }\end{array}$ & - & $\begin{array}{l}\text { Lahmann et al. (2007) 14, } \\
\text { Schimidt et al. (2008) 15, } \\
\text { Peters et al. (2010) 17, } \\
\text { Leitzmann et al. (2008) 18, } \\
\text { Suzuki et al. (2008) 19, } \\
\text { Peplonska et al. (2008) 16 * }\end{array}$ & - \\
\hline Gordura corporal & $\begin{array}{l}\text { Pré-menopausa: } \\
\text { fator provável de proteção }\end{array}$ & $\begin{array}{c}\text { Mathew et al. (2008) 20, } \\
\text { Bessaoud \& Daurès (2008) 8* }\end{array}$ & $\begin{array}{c}\text { Nemesure et al. (2009) } 12 * \\
\text { Palmer et al. (2007) } 25, \\
\text { Cust et al. (2009) } 24 \text { * }\end{array}$ & \\
\hline & $\begin{array}{l}\text { Pós-menopausa: fator } \\
\text { convincente de risco }\end{array}$ & $\begin{array}{c}\text { Ahn et al. (2009) 21, } \\
\text { Borquist et al. (2009) 22, } \\
\text { Setiawan et al. (2009) 23**, } \\
\text { Bessaoud \& Daurès (2008) 8* }\end{array}$ & $\begin{array}{l}\text { Nemesure et al. (2009) } 12 \text { *, } \\
\text { Palmer et al. (2007) } 25, \\
\text { Cust et al. (2009) } 24 * \\
\text { Setiawan et al. (2009) } 23 \text { ** }\end{array}$ & Mathew et al. (2008) 20 \\
\hline Gordura abdominal & $\begin{array}{c}\text { Pré-menopausa: } \\
\text { não há especificação } \\
\text { Pós-menopausa: } \\
\text { fator provável de risco }\end{array}$ & $\begin{array}{l}\text { Mathew et al. (2008) 20, } \\
\text { Ahn et al. (2009) 21, } \\
\text { Borquist et al. (2009) } 22\end{array}$ & - & $\begin{array}{l}\text { Mathew et al. (2008) 20, } \\
\text { Palmer et al. (2007) } 25 \\
\text { Palmer et al. (2007) } 25\end{array}$ \\
\hline $\begin{array}{l}\text { Ganho de peso } \\
\text { na vida adulta }\end{array}$ & $\begin{array}{c}\text { Pré-menopausa: os estudos } \\
\text { ainda são limitados e sem } \\
\text { conclusão }\end{array}$ & - & - & $\begin{array}{c}\text { Palmer et al. (2007) 25, } \\
\text { Wu et al. (2007) } 30\end{array}$ \\
\hline & $\begin{array}{l}\text { Pós-menopausa: fator } \\
\text { provável de risco }\end{array}$ & $\begin{array}{l}\text { Wu et al. (2007) 30, } \\
\text { Ahn et al. (2009) } 21\end{array}$ & - & Palmer et al. (2007) 25 \\
\hline $\begin{array}{l}\text { Altura atingida até } \\
\text { a idade adulta }\end{array}$ & $\begin{array}{l}\text { Pré-menopausa: fator } \\
\text { provável de risco } \\
\text { Pós-menopausa: fator } \\
\text { convincente de risco }\end{array}$ & Mathew et al. (2008) 20 & $\begin{array}{l}- \\
- \\
-\end{array}$ & Mathew et al. (2008) 20 \\
\hline Bebida alcoólica & $\begin{array}{l}\text { Fator convincente de risco } \\
\text { tanto na pré-menopausa } \\
\text { quanto na pós-menopausa }\end{array}$ & $\begin{array}{l}\text { Deandrea et al. (2008) 35, } \\
\text { Zang et al. (2007) 36, } \\
\text { Setiawan et al. (2009) 23, } \\
\text { Bestad et al. (2008) 37, } \\
\text { Duffy et al. (2009) } 38\end{array}$ & Bessaoud \& Daurés (2008) 8 & $\begin{array}{l}\text { Brown et al. (2010) 39, } \\
\text { Terry et al. (2007) } 40\end{array}$ \\
\hline
\end{tabular}

AICR: American Institute for Cancer Research; WCRF: World Cancer Research Fund.

* Nestes estudos, não foi feita a análise segundo o status da menopausa;

** A associação com o câncer de mama varia segundo os receptores hormonais. 
O efeito protetor da amamentação pode estar associado à diferenciação completa das células mamárias e ao menor tempo de exposição à ação de hormônios sexuais, que se encontram diminuídos durante a amenorréia induzida pela lactação. Além disso, a intensa esfoliação do tecido mamário e a apoptose maciça de células epiteliais, decorrentes da amamentação, podem reduzir o risco de câncer de mama por meio da eliminação de células que tenham sofrido algum dano potencial no DNA ${ }^{1}$.

\section{- Atividade física}

A prática regular de atividade física é considerada pelo WCRF e AICR 1 como fator de proteção provável para o câncer de mama na pós-menopausa. Porém, as evidências do efeito protetor desta variável ainda são limitadas para mulheres na prémenopausa (Tabela 2).

Sprague et al. 13, em um estudo do tipo casocontrole realizado nos Estados Unidos com uma amostra de 1.689 casos de câncer de mama in situ, 6.391 casos invasivos e 7.630 controles, avaliaram a atividade física no lazer, no trabalho e a atividade física total, segundo o tipo de câncer (invasivo ou in situ). Observou-se o efeito protetor somente para atividade física no lazer acima de 6 horas/semana e para o câncer de mama invasivo, tomando-se como referência o sedentarismo (OR = 0,77; IC95\%: 0,65-0,92).

Lahmann et al. 14, em um estudo de coorte desenvolvido na Europa com 218.169 mulheres, avaliaram a atividade física no lazer, no ambiente doméstico e a atividade física total, segundo o status menopausal das participantes da pesquisa. O nível de atividade física foi dividido em categorias, segundo os valores de MET (múltiplos do consumo de oxigênio em repouso), estimados a partir de valores pré-estabelecidos em MET/ hora para cada tipo de atividade física. O efeito protetor foi encontrado em mulheres na pré- e na pós-menopausa. Em mulheres na pré-menopausa, o maior quartil de prática de atividade física doméstica esteve associado com a redução no risco de câncer de mama, comparando-se com o menor quartil (RR = 0,71; IC95\%: 0,55-0,90). Para as mulheres na pós-menopausa, os maiores quartis de prática de atividade física doméstica (RR = 0,81; IC95\%: 0,70-0,93) e de atividade física total (doméstica e no lazer - RR = 0,86; IC95\%: 0,73-0,95) estiveram associados com a redução do risco da neoplasia maligna de mama, tomando-se como referências os menores quartis.

Schimidt et al. 15, em um estudo de casocontrole realizado na Alemanha com mulheres na pós-menopausa, sendo 3.414 com câncer de mama e 5.569 controles, estudaram a prática de atividade física no lazer, no trabalho e no ambiente doméstico, segundo o status dos receptores hormonais, a característica do tumor (in situ ou invasivo) e o período da vida de prática de exercício físico. Observou-se efeito protetor somente da prática de atividade física no lazer após os 50 anos de idade para o câncer de mama invasivo e receptor positivo de estrógeno e progesterona (ER/PR+). Encontrou-se OR = 0,71 (IC95\%: 0,60-0,85) para a prática de atividade física no lazer após os 50 anos de idade para tumores ER/PR+, comparando-se o maior e o menor quintis de atividade física. Este resultado sugere que a atividade física no lazer após os 50 anos parece ser mais efetiva na prevenção do câncer de mama do que antes desta idade. Esta evidência também foi comprovada por Peplonska et al. 16, na Polônia, com 2.176 casos e 2.326 controles. $\mathrm{Na}$ referida pesquisa, a prática de atividade física, com exceção do exercício físico no trabalho, mostrou-se protetora para o câncer de mama em todas as fases da vida, em especial a partir dos 50 anos de idade. Nesta faixa etária, encontrou-se um valor de OR = 0,69 (IC95\%: 0,54-0,89) para o maior tercil de prática de atividade física no lazer (moderada ou vigorosa), comparando-se com a ausência deste tipo de atividade. Neste estudo, os níveis de atividade física foram definidos segundo os valores de MET, sendo consideradas atividades moderada ou vigorosa aquelas cujo MET foi igual ou superior a 4,5.

Peters et al. 17, em uma coorte de 182.862 mulheres na pós-menopausa desenvolvida nos Estados Unidos, avaliaram a atividade física total (trabalho, lazer e doméstica), segundo história familiar de câncer de mama e estado nutricional. Observou-se que a prática de exercício físico igual ou superior a três vezes/semana exerceu efeito protetor para a neoplasia maligna de mama (RR = 0,93; IC95\%: 0,87-1,0), tomando-se como referência a inatividade. $\mathrm{Na}$ análise segundo o estado nutricional e o histórico familiar de neoplasia maligna de mama, detectou-se o efeito protetor para mulheres com sobrepeso (índice de massa corporal - IMC $\geq 25 \mathrm{~kg} / \mathrm{m}^{2}$ ), e em mulheres com e sem história familiar de câncer de mama, sendo a relação mais forte no primeiro caso. O resultado referente ao estado nutricional foi comprovado por Peplonska et al. 16, e foi oposto ao observado por Leitzmann et al. 18. Segundo Peplonska et al. 16, o efeito protetor da prática de atividade física total (trabalho, lazer e doméstica) restringiu-se às mulheres cujo IMC $\geq 25 \mathrm{~kg} / \mathrm{m}^{2}$ (OR = 0,68; IC95\%: 0,54-0,85), comparando-se o maior e o menor quartis de atividade física, em MET/hora/semana. Segundo estes autores, mulheres mais pesadas tendem a apresentar mais benefícios com a prática regular 
de exercícios físicos, visto que nessas, a prática de exercícios provoca queda mais significativa nos níveis séricos de fatores pró-inflamatórios. Leitzmann et al. 18, em uma coorte nos Estados Unidos com 32.269 mulheres na pós-menopausa, observaram efeito protetor da atividade física total - trabalho, lazer e doméstica $(R R=0,81$; IC95\%: 0,69-0,95) e da atividade física vigorosa (RR = 0,82; IC95\%: 0,70-0,96), comparando-se o maior e o menor quintis, em MET/hora/semana. Este efeito restringiu-se às mulheres com IMC < $25 \mathrm{~kg} / \mathrm{m}^{2}$. Foram consideradas como vigorosas as seguintes atividades: limpar o chão, capinar, cortar lenha, correr, pedalar rápido, jogar tênis, praticar ginástica e dança extenuantes, entre outras. Ainda neste estudo, a relação entre atividade física e câncer de mama não variou segundo o status dos receptores hormonais.

Suzuki et al. 19, em uma coorte no Japão com 30.157 mulheres, encontraram um valor de $\mathrm{RR}=$ 0,45 (IC95\%: 0,25-0,78), comparando-se a categoria mais ativa ( $\geq 1 \mathrm{~h} /$ semana de caminhada e $\geq 1 \mathrm{~h}$ /semana de outro exercício) com a menos ativa $(<1 \mathrm{~h} /$ semana de caminhada e $<1 \mathrm{~h} /$ semana de outro exercício). Este efeito protetor não foi modificado segundo o status da menopausa e o estado nutricional, o que sugere que a atividade física exerça papel protetor independentemente dos aspectos acima relacionados.

Os dados relativos à associação entre exercício físico e câncer de mama ainda são inconsistentes no que se refere aos diferentes tipos de atividade física (modalidade, intensidade, frequência), aos períodos de vida em que ocorreu a prática de exercício físico, ao status da menopausa, ao estado nutricional, aos antecedentes pessoais e às características dos tumores. Isto decorre principalmente da falta de padronização das técnicas de mensuração e de classificação do nível de atividade física, conforme pode ser observado na descrição metodológica dos estudos mencionados anteriormente. Além disso, há dificuldade em se estimar o nível de exercício físico, uma vez que se trata de um dado referido e que depende da memória e da precisão das respostas do entrevistado. Assim, a falta de padronização da metodologia limita a comparação dos resultados e a elucidação das evidências.

Apesar de todas as questões ainda pendentes, a prática regular de atividade física provavelmente exerce um efeito protetor para a neoplasia maligna de mama, visto que pode promover o atraso da menarca, uma maior quantidade de ciclos anovulatórios e irregulares, a redução do estrógeno sérico, o aumento de globulinas que se ligam a hormônios sexuais, a redução do processo inflamatório, a melhora da função imune, além de auxiliar no controle de peso e melhorar a sen- sibilidade à ação da insulina 13,18. Alguns autores também levantam a hipótese de que a atividade física pode modular a expressão de receptores de estrógeno e de progesterona (ER/PR). Mulheres mais ativas tendem a ter maior quantidade de globulinas que se ligam a receptores hormonais, menor concentração sérica de estrógeno, e, consequentemente, menor expressão de ER/PR, uma vez que esses genes são ativados em parte pelo estrogênio 15,17 .

\section{Fatores de risco para o câncer de mama}

\section{- Gordura corporal}

A gordura corporal é apontada pelo WCRF e AICR ${ }^{1}$ como fator de risco convincente para o câncer de mama em mulheres na pós-menopausa, e como fator de proteção provável para as mulheres na pré-menopausa. Em estudos recentes, tem-se observado que os resultados encontrados, tanto para mulheres na pré-menopausa quanto na pós-menopausa, são controversos (Tabela 2). Mathew et al. 20, em um estudo realizado na Índia com uma amostra de 1.866 casos e 1.873 controles, detectaram que o IMC $\geq 30 \mathrm{~kg}$ / $\mathrm{m}^{2}$ foi considerado um fator de risco para câncer de mama na pré-menopausa $(\mathrm{OR}=1,56$; IC95\%: 1,03-2,35), considerando como referência o IMC de mulheres eutróficas $\left(<25 \mathrm{~kg} / \mathrm{m}^{2}\right)$. Neste mesmo estudo, não foi encontrada associação significativa entre o IMC e o câncer de mama em mulheres na pós-menopausa.

Em uma coorte de 99.039 mulheres na pósmenopausa 21, nos Estados Unidos, observou-se um aumento no risco de câncer de mama a partir do IMC $=25 \mathrm{~kg} / \mathrm{m}^{2}$, sendo que, para o IMC $\geq$ $40 \mathrm{~kg} / \mathrm{m}^{2}$, o valor de RR foi de 2,08 (IC95\%: 1,44$2,99)$, quando comparado ao IMC de referência $\left(18,5 \mathrm{~kg} / \mathrm{m}^{2}\right.$ a $\left.22,4 \mathrm{~kg} / \mathrm{m}^{2}\right)$. Resultados semelhantes foram encontrados por Borquist et al. $22 \mathrm{e}$ Bessaoud \& Daurès 8. Borquist et al. 22 , em uma coorte de 9.685 mulheres suecas na pós-menopausa, observaram que o aumento das medidas antropométricas, em especial o IMC, esteve associado às formas menos agressivas de câncer de mama. Encontrou-se um valor de $\mathrm{RR}=1,53$ (IC95\%: 1,05-2,24; $\mathrm{p}<0,01$ ) para o maior quartil de IMC, comparando-se ao menor quartil. Bessaoud \& Daurès 8 realizaram um estudo na França com 437 casos e 922 controles, porém, não analisaram as variáveis segundo o status da menopausa. Como resultado, obtiveram um valor de OR = 1,90 (IC95\%: 1,26-2,86) para um IMC > $30 \mathrm{~kg} / \mathrm{m}^{2}$, tomando-se como referência o IMC entre $18 \mathrm{~kg} / \mathrm{m}^{2}$ e $25 \mathrm{~kg} / \mathrm{m}^{2}$.

Setiawan et al. 23, nos Estados Unidos, em uma coorte de 84.427 mulheres, obtiveram um RR 
de 1,53 (IC95\%: 1,29-1,81) para mulheres na pósmenopausa com IMC $\geq 30 \mathrm{~kg} / \mathrm{m}^{2}$, comparandose às mulheres com $\mathrm{IMC}<25 \mathrm{~kg} / \mathrm{m}^{2}$, porém, este resultado foi observado apenas em mulheres com câncer de mama ER/PR+. Para mulheres na pósmenopausa, mas com os receptores hormonais negativos, o IMC $\geq 30 \mathrm{~kg} / \mathrm{m}^{2}$ mostrou-se como fator protetor para o câncer de mama, tendo como referência também mulheres com IMC $<25 \mathrm{~kg} / \mathrm{m}^{2}$ (RR = 0,69; IC95\%: 0,49-0,98). Os resultados deste estudo sugerem que os receptores hormonais podem modificar os riscos existentes entre excesso de gordura corporal e câncer de mama.

Nemesure et al. 12 e Cust et al. 24 observaram efeito protetor do excesso de peso para o câncer de mama, porém, não realizaram as análises segundo o status da menopausa. No primeiro estudo, observou-se um valor de OR = 0,48 (IC95\%: 0,290,80 ), comparando-se o maior e o menor quintis de IMC. No estudo de Cust et al. 24, encontrou-se o mesmo valor de OR, porém, comparando-se o maior e o menor tercis de IMC (OR = 0,48; IC95\%: 0,30-0,78). Estes resultados estão de acordo com os de Palmer et al. ${ }^{25}$, que estudaram uma coorte de 59 mil mulheres dos Estados Unidos e fizeram as análises segundo o status da menopausa. Estes autores constataram que, tanto em mulheres na pré-menopausa quanto na pós-menopausa, o excesso de gordura corporal aos 18 anos de idade e no momento da pesquisa, apresentaram efeito protetor para a neoplasia maligna de mama. Para o câncer de mama na pré-menopausa, este resultado é compatível com os achados do WCRF e AICR 1, o que não acontece com a neoplasia na pós-menopausa.

Bardia et al. 26 consideraram o peso aos 12 anos de idade e o câncer de mama na pós-menopausa, pois existem hipóteses de que o maior IMC na adolescência seja um fator protetor para o câncer de mama tanto na pré-menopausa como na pós-menopausa. O mecanismo é desconhecido, mas se sabe que maiores níveis de insulina e IGF-1 na adolescência podem prejudicar o metabolismo ovariano, induzindo à anovulação. Bardia et al. 26 encontraram um RR de 0,85 (IC95\%: 0,74-0,98) somente para mulheres que possuíam o peso aos 12 anos acima da média para a idade, comparando-se àquelas com peso na média para a respectiva idade.

Embora o IMC não seja uma medida direta de avaliação da composição corporal, estudos epidemiológicos comumente usam-no como indicador de excesso de gordura corporal, visto que há uma forte correlação ( $r$ varia de 0,72 a 0,84 ) entre este índice e o percentual de gordura corpórea 27.

Alguns mecanismos são apontados para explicar a relação entre a gordura corporal e o ris- co de câncer de mama na pós-menopausa. $\mathrm{O}$ excesso de peso promove o aumento do nível de estrógeno circulante, pois o tecido adiposo constitui o principal local de síntese de estrógeno em mulheres na pós-menopausa, e promove o aumento da insulina e do fator de crescimento semelhante à insulina (IGF-1) circulantes. Estas substâncias, juntamente com outros fatores pró-inflamatórios, como o fator de necrose tumoral (TNF), interleucina e proteína $\mathrm{C}$ reativa, produzidos pelos adipócitos, induzem à progressão do ciclo celular e à inibição da apoptose, aumentando, assim, o risco de desenvolvimento do câncer 1 . Além disso, acredita-se que estes fatores mencionados promovam a superexpressão de oncogenes 28.

Em relação às mulheres na pré-menopausa, não existe um mecanismo bem esclarecido que explique a gordura corporal como fator de proteção. Existe a hipótese de que o excesso de peso poderia levar a ciclos anovulatórios mais frequentes e, assim, reduzir a exposição à progesterona endógena 1. Segundo Palmer et al. 25, existem evidências de que o estradiol é depurado no fígado de forma mais rápida em mulheres jovens e com excesso de peso. Há a hipótese também de que mulheres magras podem estar mais expostas biologicamente aos carcinogênicos. Além disso, Wolf \& Anderson 29 sugerem que mulheres obesas, por possuírem maior reserva de gordura, apresentam menores níveis séricos de toxinas, pois as mesmas são sequestradas no tecido adiposo.

\section{- Gordura abdominal}

De acordo com o WCRF e AICR 1 , a gordura abdominal é definida como fator de risco provável para a neoplasia maligna de mama somente na pós-menopausa. Estas evidências foram confirmadas em estudos analíticos recentes 20,21,22.

Segundo Mathew et al. 20, a circunferência da cintura (CC) aumentada ( $>85 \mathrm{~cm}$ ) constitui um fator de risco para o câncer de mama em mulheres na pós-menopausa (OR = 1,61; IC95\%: 1,22$2,12)$, porém, esta associação não foi significativa na pré-menopausa. Ahn et al. 21, em uma coorte de 99.039 mulheres na pós-menopausa, também observaram que a $\mathrm{CC} \geq 97 \mathrm{~cm}$ elevou o risco de câncer de mama. Conforme o estudo, mulheres com CC $>103 \mathrm{~cm}$ apresentaram $55 \%$ mais chance de desenvolverem câncer de mama do que aquelas que possuíam esta medida igual ou inferior a 75cm (RR = 1,55; IC95\%: 1,16-2,06). Resultado semelhante foi encontrado por Borquist et al. ${ }^{22}$, em uma coorte de 9.685 mulheres na pós-menopausa, em que se observou um valor de $\mathrm{RR}=$ 1,93 (IC95\%: 1,29-2,89) para o maior quartil de 
circunferência da cintura, comparando-se com o menor quartil desta variável.

\section{- Ganho de peso na idade adulta}

O ganho de peso ao longo da vida adulta é considerado pelo WCRF e AICR ${ }^{1}$ como um fator de risco provável apenas para mulheres na pós-menopausa, enquanto que, na pré-menopausa, as evidências são inconclusivas (Tabela 2).

Em estudos recentes 21,25,30, o ganho de peso corporal ao longo da idade adulta tem sido evidenciado como fator de risco para a neoplasia maligna de mama. Segundo Ahn et al. 21, em uma coorte desenvolvida nos Estados Unidos com quase cem mil mulheres na pós-menopausa, o ganho de peso igual ou acima de $20 \mathrm{~kg}$ ao longo da idade adulta aumentou o risco de neoplasia maligna de mama. Mulheres que engordaram de 20 a $30 \mathrm{~kg}$, considerando-se a diferença entre o peso corporal no momento da pesquisa e o peso aos 18 anos de idade, apresentaram um valor de $R R=1,56$ (IC95\%: 1,10-2,21), quando comparadas àquelas que mantiveram o peso relativamente estável (variação de $\pm 2 \mathrm{~kg}$ ). Para mulheres que perderam peso, não houve associação significativa. Este estudo também avaliou o ganho de peso em estágios diferentes da vida. $\mathrm{O}$ aumento de peso ocorrido a partir de 50 anos até o momento da pesquisa, e entre os 35 e 50 anos representaram aumento do risco para a o câncer de mama na pós-menopausa. Observou-se ainda que a associação entre ganho de peso e câncer de mama é mais forte quando a idade da menarca é mais tardia, e quando a mulher não faz uso de terapia de reposição hormonal (TRH). A explicação para tais fatos é que o efeito do ganho de peso é mascarado pela menarca precoce, na qual há maior quantidade de estrógeno acumulado, e que a TRH promove o aumento do estrógeno circulante, mascarando também os efeitos da adiposidade.

Resultado semelhante foi encontrado por Wu et al. ${ }^{30}$, em uma amostra de 1.277 casos e 1.160 controles, em que foi encontrado um valor de OR de 1,66 (IC95\%: 1,09-2,53) para a categoria de ganho de peso superior a $20 \mathrm{~kg}$, comparando-se àquelas cujo ganho de peso na vida adulta foi igual ou inferior a $10 \mathrm{~kg}$, para o câncer de mama na pós-menopausa. Na pré-menopausa, as associações entre o ganho de peso e o câncer de mama não foram significativas. Palmer et al. 25 também não encontraram associações significativas entre ganho de peso ao longo da idade adulta e câncer de mama, tanto na pré-menopausa quanto na pós-menopausa.

A quantidade de peso ganho e sua associação com o câncer de mama ainda não está clara.
Ainda também não está bem definido se a associação entre neoplasia maligna de mama e o ganho de peso é diferente em períodos específicos da vida 31 . Segundo Ahn et al. 21 , esta relação é mais evidente considerando-se o ganho de peso total - diferença entre o peso do momento da pesquisa e o peso aos 18 anos - do que quando se limitam tempos específicos da vida. A mesma tendência foi relatada por Lahmann et al. ${ }^{32}$. Estes autores usaram como metodologia para verificação do ganho de peso a diferença entre o peso no momento da entrevista e o peso aos 20 anos de idade, e discutem que poucos estudos foram capazes de verificar as variações de peso em diferentes idades da vida, e que, nestes casos, não houve efeitos diferentes no risco de câncer de mama.

Os mecanismos da gordura abdominal, bem como do ganho de peso envolvidos na carcinogênese mamária são semelhantes aos mecanismos do excesso de gordura corporal, que já foram mencionados anteriormente.

\section{- Estatura}

A estatura atingida até a idade adulta é considerada pelo WCRF e AICR ${ }^{1}$ como um fator de risco provável na pré-menopausa, e convincente na pós-menopausa. Mathew et al. 20 realizaram um estudo na Índia, com 1.866 casos e 873 controles, e verificaram um valor de OR de 1,61 (IC95\%: $1,08-2,42)$ para a categoria de mulheres que possuíam $160 \mathrm{~cm}$ de altura ou mais, comparando-se com aquelas cuja altura era inferior a $160 \mathrm{~cm}$, em mulheres na pós-menopausa. Já para mulheres na pré-menopausa, as associações não foram significativas.

Li et al. 33 e Beaber et al. 6 também fizeram a análise da estatura, porém, considerando a idade em que as mulheres tinham alcançado a altura máxima. Li et al. 33 avaliaram uma coorte dos Estados Unidos, composta de 27.536 mulheres na pós-menopausa, e verificaram um risco $1,5 \mathrm{vez}$ maior de câncer de mama para aquelas que atingiram a altura máxima aos 12 anos de idade ou menos (RR = 1,5; IC95\%: 1,1-2,0), comparando-se com aquelas cuja altura máxima foi atingida aos 17 anos ou mais, e este resultado foi restrito ao câncer de mama invasivo. Resultado semelhante foi encontrado por Beaber et al. 6 que, em um estudo caso-controle realizado nos Estados Unidos com mulheres na pós-menopausa, obtiveram um valor de OR = 1,6 (IC95\%: 1,0-2,4) para aquelas que alcançaram a altura máxima aos 14 anos ou menos, tomando-se como referência aquelas cuja altura máxima foi atingida aos 17 anos ou mais. Este resultado foi restrito ao câncer de mama do tipo ductal-lobular. 
O maior ganho de estatura obtido até a idade adulta pode estar relacionado a muitos processos carcinogênicos, por envolver a atuação do hormônio do crescimento, do fator de crescimento semelhante à insulina e de hormônios sexuais. Acredita-se que mulheres mais altas tenham sido submetidas a mais divisões celulares estimuladas por hormônios, havendo, portanto, maior probabilidade de ocorrência de erros durante a replicação do DNA ${ }^{34}$. Existe também a hipótese de que a exposição precoce do tecido mamário aos altos níveis de hormônio do crescimento e ao fator de crescimento semelhante à insulina, que acontece quando a pessoa cresce rapidamente, pode contribuir para a ocorrência do câncer de mama 33 .

\section{- Bebida alcoólica}

Segundo o WCRF e AICR 1, a ingestão de bebida alcoólica representa um fator de risco convincente para o câncer de mama, tanto em mulheres na pré-menopausa quanto na pós-menopausa. Estudos recentes 23,35,36,37,38 confirmam este efeito de risco da bebida alcoólica. Setiawan et al. ${ }^{23}$, em uma coorte de 84.427 mulheres, avaliaram a associação entre ingestão de bebida alcoólica e câncer de mama, segundo o status dos receptores hormonais. Detectou-se um valor de RR de 1,40 (IC95\%: 1,14-1,72) para a ingestão de bebida alcoólica aumentada ( $\geq 2$ doses/dia), comparando-se com a não ingestão de bebida alcoólica, para o câncer de mama ER/PR+. Já para o câncer do tipo ER/PR-, o valor de RR encontrado foi de 1,71 (IC95\%: 1,19-2,46). Estes valores de RR não foram diferentes estatisticamente ( $p=0,07)$, indicando que o status dos receptores hormonais não modificou o risco. Além disso, como o álcool também esteve associado aos receptores negativos, sugere-se que ele atue por meio de outros mecanismos além dos hormonais.

Deandrea et al. 35, em um estudo caso-controle na Itália, constataram que a ingestão aumentada de bebida alcoólica ( $\geq 13,8 \mathrm{~g} /$ dia) elevou o risco de câncer de mama, quando se comparou com mulheres que nunca fizeram uso de bebida alcoólica (OR = 1,96; IC95\%: 1,57-2,47). Porém, quando foi realizada a análise segundo o status dos receptores, o efeito de risco foi observado apenas para receptores de estrógeno positivos. Segundo estes autores, o risco para câncer de mama decorrente do uso de bebidas alcoólicas está mais relacionado com receptores de estrógeno positivos do que negativos. Por meio de estudos in vitro, observou-se atividade específica do etanol em células mamárias humanas com receptores positivos, mas não com receptores negativos de estrógeno 35,36.
Zang et al. 36, em uma coorte nos Estados Unidos com 38.454 mulheres, também observaram efeito de risco da ingestão de bebida alcoólica para os cânceres de mama in situ, invasivo e ER/ PR+. O aumento de $10 \mathrm{~g}$ no consumo de etanol por dia provocou um aumento de $7 \%$ no risco de desenvolvimento do câncer de mama in situ (RR = 1,07; IC95\%: 1,01-1,14), e de 9\% no risco de câncer de mama invasivo (RR = 1,09; IC95\%: 1,02$1,16)$. Na análise segundo o status dos receptores hormonais, verificou-se risco aumentado somente para o câncer de mama ER/PR+, para o aumento no consumo de $10 \mathrm{~g}$ de etanol diário (RR = 1,11; IC95\%: 1,03-1,20). Vale ressaltar que o aumento do risco foi verificado apenas para o consumo de cerveja, e isto pode ser explicado por ser o tipo de bebida alcoólica mais consumido naquela amostra.

Bestad et al. 37, em um estudo caso-controle realizado nos Estados Unidos, fizeram a análise do consumo de bebida alcoólica segundo o tipo de bebida e a época de ingestão. Observaram associação significativa apenas para a ingestão aumentada de bebida alcoólica ( $\geq 2$ drinques/ dia) nos últimos 5 anos antes da entrevista (OR = 1,82; IC95\%: 1,01-3,28), não sendo encontrada associação para a ingestão ao longo de toda a vida adulta (dos 15 anos até a data da entrevista) e na juventude (dos 15 aos 20 anos). Com este resultado, podem surgir duas explicações: ou o efeito do álcool no risco de câncer de mama não se acumula ao longo do tempo, ou o resultado pode ser decorrente do viés de memória, uma vez que as mulheres tendem a se lembrar melhor da ingestão recente. No que se refere aos diferentes tipos de bebidas alcoólicas, não foram encontradas diferenças significativas entre elas, o que revela que o etanol, independentemente do tipo de bebida em que é veiculado, causa aumento no risco de tal neoplasia 36,37 .

Seguindo a mesma tendência, Duffy et al. ${ }^{38}$, em uma coorte dos Estados Unidos composta de 88.530 mulheres na pós-menopausa, verificaram que, até mesmo a baixa ingestão de bebida alcoólica (5,6g de etanol/dia) foi considerada um fator de risco para a neoplasia maligna de mama $(\mathrm{RR}=$ 1,005; IC95\%: 1,001-1,009).

Em contradição com os resultados apresentados, Bessaoud \& Daurès 8 , em um estudo de casocontrole desenvolvido na França, verificaram que a ingestão de uma dose diária de bebida alcoólica (10-15g de etanol/dia) foi considerada um fator de proteção para o câncer de mama, quando se compara com as mulheres que não fazem o uso de bebida alcoólica (OR = 0,21; IC95\%: 0,10-0,91). Este efeito protetor foi encontrado tanto para a ingestão moderada ( $<1$ dose/dia) de vinho (OR = 0,51; IC95\%: 0,30-0,94) quanto para as demais 
bebidas, $(\mathrm{OR}=0,63$; IC95\%: 0,42-0,94). Com base nestes resultados, sugere-se que exista um limiar de ingestão de bebida alcoólica, abaixo do qual a mesma atua como fator protetor e, acima, como fator de risco. No que se refere ao efeito protetor do vinho, especificamente, sugere-se que o efeito deletério do álcool seja, em parte, atenuado pela presença de agentes antioxidantes presentes no vinho, quando sua ingestão é moderada ${ }^{8}$.

Apesar de todas as associações de proteção e de risco relacionadas ao consumo de bebida alcoólica, Brown et al. 39 e Terry et al. ${ }^{40}$, em estudos caso-controle, não encontraram associações significativas entre esta variável e o câncer de mama. Esta falta de associação foi explicada em parte pela homogeneidade no consumo de bebida alcoólica e pelo baixo consumo dos mesmos entre as mulheres da amostra. Além dos achados contraditórios, os dados ainda são inconsistentes no que se refere à associação entre câncer de mama e ingestão de bebida alcoólica, segundo o status dos receptores hormonais, o tipo e a quantidade de bebida alcoólica ingerida e o período de exposição ao álcool 8,36,37.

Apesar das controvérsias, existem muitas evidências de que o álcool pode aumentar o risco de câncer de mama por meio de diversos mecanismos, dependentes ou não de hormônios. $\mathrm{O}$ etanol pode agir como cocarcinogênico, aumentando a permeabilidade da membrana celular a carcinógenos, inibindo a detoxificação dos mesmos pelo fígado, prejudicando o metabolismo de nutrientes e induzindo ao estresse oxidativo. Além disso, pode atuar como mutagênico, por meio do acetaldeído, e pode aumentar os níveis séricos de estrógenos e a atividade de transcrição do receptor de estrógeno, elevando a resposta da célula à ação deste hormônio 1,35,36,40.

\section{Conclusões}

A partir desta revisão sistemática, pode-se chegar às seguintes conclusões: (1) a lactação, bem como a prática de atividade física são fatores protetores para o câncer de mama, tanto na pré- quanto na pós-menopausa; (2) os resultados referentes à gordura corporal são contraditórios, na pré- e na pós-menopausa, embora o WCRF e o AICR tenham considerado o excesso de gordura corporal na pós-menopausa como fator de risco convincente; (3) o aumento das medidas antropométricas (circunferência da cintura, peso ao longo da vida adulta e estatura) representa fator de risco para o câncer de mama na pós-menopausa; (4) a ingestão de bebida alcoólica representa fator de risco para esta enfermidade, na pré- e na pósmenopausa, segundo a maior parte dos estudos. Assim, pode-se dizer que as evidências estabelecidas pelo WCRF e pelo AICR estão sendo confirmadas para todas as variáveis analisadas neste estudo, com exceção da gordura corporal.

Apesar dos achados, muitas questões envolvidas na prevenção da carcinogênese mamária ainda precisam ser elucidadas por meio de estudos bem delineados. É preciso definir: (1) o tempo mínimo de lactação que protege contra o câncer de mama; (2) a quantidade, intensidade e o período de vida em que a atividade física exerce papel protetor, visto que as informações ainda são muito heterogêneas em relação a estes aspectos; (3) a quantidade de peso corporal ganho e o período da vida (fase adulta) em que o risco é aumentado; (4) o tipo e a quantidade de bebida alcoólica associados ao risco de câncer de mama.

Com base nas informações atuais, a melhor forma de prevenção primária do câncer de mama, considerando os fatores investigados nesta revisão sistemática, é a amamentação e a adoção de um estilo de vida saudável, incluindo a prática regular de atividade física, a manutenção de peso corporal adequado e o consumo moderado ou ausente de álcool. 


\section{Resumo}

O objetivo desta revisão foi investigar alguns fatores de risco e de proteção para câncer de mama e analisar se as evidências científicas estabelecidas pelo World Cancer Research Fund e American Institute for Cancer Research e publicadas em 2007 foram confirmadas por estudos mais recentes. Em maio de 2010 foi realizada uma revisão sistemática de estudos coorte e caso-controle publicados entre 2007 e 2010, nas bases PubMed, LILACS e SciELO. Foram selecionados 27 artigos, sendo 13 caso-controles e 14 coortes. As práticas de lactação e de atividade física constituem fatores de proteção para o câncer de mama, e o consumo de bebida alcoólica, fator de risco. Observa-se relação diretamente proporcional entre o aumento da circunferência da cintura, do peso ao longo da vida adulta e da estatura, e risco de câncer de mama na pós-menopausa. A associação entre gordura corporal e câncer de mama é contraditória, na pré- e na pós-menopausa. Até o presente momento, é possível inferir que a melhor forma de prevenção do câncer de mama é a amamentação e a adoção de um estilo de vida saudável.

Neoplasias da Mama; Antropometria; Consumo de Bebidas Alcoólicas; Atividade Física; Lactação

\section{Referências}

1. World Cancer Research Fund/American Institute for Cancer Research. Food, nutrition, physical activity, and the prevention of cancer: a global perspective. Washington DC: American Institute for Cancer Research; 2007.

2. Adami HO, Day NE, Trichopoulos D, Willett WC. Primary and secondary prevention in the reduction of cancer morbidity and mortality. Eur J Cancer 2001; 37 Suppl 8:S118-27.

3. Instituto Nacional de Câncer. Estimativa 2010: incidência de câncer no Brasil. Rio de Janeiro: Instituto Nacional de Câncer; 2009.

4. Graham AC. Epidemiology and prevention of breast cancer. Cancer Epidemiol Biomarkers Prev 2005; 14:768-72.

5. Huo D, Adebamowo CA, Ogundiran TO, Akang EE, Campbell O, Adenipekun A, et al. Parity and breastfeeding are protective against breast cancer in Nigerian women. Br J Cancer 2008; 98:992-6.

6. Beaber EF, Holt VL, Malone KE, Porter PL, Daling JR, Li CI. Reproductive factors, age at maximum height, and risk of three histologic types of breast cancer. Cancer Epidemiol Biomarkers Prev 2008; $17: 3427-34$

\section{Colaboradores}

M. M. V. Naves contribuiu na concepção do trabalho (idéia e planejamento), na redação e na revisão crítica do manuscrito, bem como aprovou a versão a ser publicada. L. E. Inumaru contribuiu na revisão da literatura, na análise e na síntese dos dados, na redação do artigo, e aprovou a versão a ser publicada. E. A. Silveira auxiliou na concepção e na redação do trabalho e aprovou a versão a ser publicada.

\section{Agradecimentos}

À Pró-Reitoria de Pesquisa e Pós-graduação da Universidade Federal de Goiás (PRPPG-UFG) pela concessão de bolsa de estudos (mestrado).
7. Stuebe AM, Willett WC, Xue F, Michels KB. Lactation and incidence of premenopausal breast cancer: a longitudinal study. Arch Intern Med 2009; 169:1364-71.

8. Bessaoud F, Daurès JP. Patterns of alcohol (especially wine) consumption and breast cancer risk: a case-control study among a population in Southern France. Ann Epidemiol 2008; 18:467-75.

9. Gajalakshmi V, Mathew A, Brennan P, Rajan B, Kanimozhi VC, Mathews A, et al. Breastfeeding and breast cancer risk in India: a multicenter casecontrol study. Int J Cancer 2009; 125:662-5.

10. Lord SJ, Bernstein L, Johnson KA, Malone KE, McDonald JA, Marchbanks PA, et al. Breast cancer risk and hormone receptor status in older women by parity, age at first birth, and breastfeeding: a casecontrol study. Cancer Epidemiol Biomarkers Prev 2008; 17:1723-30.

11. Collaborative Group on Hormonal Factors in Breast Cancer. Breast cancer and breastfeeding: collaborative reanalysis of individual data from 47 epidemiological studies in 30 countries, including 50302 women with breast cancer and 9673 women without the disease. Lancet 2002; 360:187-95. 
12. Nemesure B, Wu SY, Hambleton IR, Leske MC, Hennis AJ. Risk factors for breast cancer in a black population - the Barbados National Cancer Study. Int J Cancer 2009; 124:174-9.

13. Sprague BL, Trentham-Dietz A, Newcomb PA, Titus-Ernstoff L, Hampton JM, Egan KM. Lifetime and occupational physical activity and risk of in situ and invasive breast cancer. Cancer Epidemiol Biomarkers Prev 2007; 16:236-43.

14. Lahmann PH, Friedenreich C, Schuit AJ, Salvini S, Allen NE, Key TJ, et al. Physical activity and breast cancer risk: the European Prospective Investigation into Cancer and Nutrition. Cancer Epidemiol Biomarkers Prev 2007; 16:36-42.

15. Schmidt ME, Steindorf K, Mutschelknauss E, Slanger T, Kropp S, Obi N, et al. Physical activity and postmenopausal breast cancer: effect modification by breast cancer subtypes and effective periods in life. Cancer Epidemiol Biomarkers Prev 2008; 17:3402-10.

16. Peplonska B, Lissowska J, Hartman TJ, SzeszeniaDabrowska N, Blair A, Zatonski W, et al. Adulthood lifetime physical activity and breast cancer. Epidemiology 2008; 19:226-36.

17. Peters TM, Schatzkin A, Gierach GL, Moore SC, Lacey Jr. JV, Wareham NJ, et al. Physical activity and postmenopausal breast cancer risk in the NIH-AARP diet and health study. Cancer Epidemiol Biomarkers Prev 2009; 18:289-96.

18. Leitzmann MF, Moore SC, Peters TM, Lacey Jr. JV, Schatzkin A, Schairer C, et al. Prospective study of physical activity and risk of postmenopausal breast cancer. Breast Cancer Res 2008; 10:R92.

19. Suzuki S, Kojima M, Tokudome S, Mori M, Sakauchi F, Fujino Y, et al. Effect of physical activity on breast cancer risk: findings of the Japan Collaborative Cohort Study. Cancer Epidemiol Biomarkers Prev 2008; 17:3396-401.

20. Mathew A, Gajalakshmi V, Rajan B, Kanimozhi V, Brennan P, Mathew BS, et al. Anthropometric factors and breast cancer risk among urban and rural women in South India: a multicentric case-control study. Br J Cancer 2008; 99:207-13.

21. Ahn J, Schatzkin A, Lacey Jr. JV, Albanes D, BallardBarbash R, Adams KF, et al. Adiposity, adult weight change, and postmenopausal breast cancer risk. Arch Intern Med 2007; 167:2091-102.

22. Borgquist S, Jirstrom K, Anagnostaki L, Manjer J, Landberg G. Anthropometric factors in relation to different tumor biological subgroups of postmenopausal breast cancer. Int J Cancer 2009; 124:402-11.

23. Setiawan VW, Monroe KR, Wilkens LR, Kolonel LN, Pike MC, Henderson BE. Breast cancer risk factors defined by estrogen and progesterone receptor status: the Multiethnic Cohort Study. Am J Epidemiol 2009; 169:1251-9.

24. Cust AE, Stocks T, Lukanova A, Lundin E, Hallmand G, Kaaks R, et al. The influence of overweight and insulin resistance on breast cancer risk and tumour stage at diagnosis: a prospective study. Breast Cancer Res Treat 2009; 113:567-76.

25. Palmer JR, Adamns-Campbell LL, Boggs DA, Wise LA, Rosenberg L. A prospective study of body size and breast cancer in black women. Cancer Epidemiol Biomarkers Prev 2007; 16:1795-802.
26. Bardia A, Vachon CM, Olson JE, Vierkant RA, Wang $\mathrm{AH}$, Hartmann LC, et al. Relative weight at age 12 and risk of postmenopausal breast cancer. Cancer Epidemiol Biomarkers Prev 2008; 17:374-8.

27. Flegal KM, Shepherd JA, Looker AC, Graubard BI, Borrud LG, Ogden CL, et al. Comparisons of percentage body fat, body mass index, waist circumference, and waist-stature ratio in adults. Am J Clin Nutr 2009; 89:500-8.

28. Barros ACSD, Barros MAC. HER e câncer de mama: inter-relações biológicas, prognósticas e terapêuticas. São Paulo: Roche; 2006.

29. Wolf MS, Anderson HA. Environmental contaminants and body fat distribution. Cancer Epidemiol Biomarkers Prev 1999; 8:179-83.

30. Wu AH, Yu MC, Tseng CC, Pike MC. Body size, hormone therapy and risk of breast cancer in AsianAmerican women. Int J Cancer 2007; 120:844-52.

31. Harvie M, Howell A, Vierkant RA, Kumar N, Cerhan JR, Kelemen LE, et al. Association of gain and loss of weight before and after menopause with risk of postmenopausal breast cancer in the Iowa Women's Health Study. Cancer Epidemiol Biomarkers Prev 2005; 14:656-61.

32. Lahmann PH, Schulz M, Hoffmann K, Boeing H, Tjønneland A, Olsen A, et al. Long-term weight change and breast cancer risk: the European Prospective Investigation into Cancer and Nutrition (EPIC). Br J Cancer 2005; 93:582-9.

33. Li CI, Littman AJ, White E. Relationship between age maximum height is attained, age at menarche, and age at first full-term birth and breast cancer risk. Cancer Epidemiol Biomarkers Prev 2007; 16:2144-9.

34. Naves MMV, Quintanilha MIGD, Inumaru LE. Nutrição e prevenção de câncer: evidências, metas de Saúde Pública e recomendações individuais. Nutrição em Pauta 2008; 16:40-5.

35. Deandrea S, Talamini R, Foschi R, Montella M, Dal Maso L, Falcini F, et al. Alcohol and breast cancer risk defined by estrogen and progesterone receptor status: a case-control study. Cancer Epidemiol Biomarkers Prev 2008; 17:2025-8.

36. Zhang SM, Lee IM, Manson JE, Cook NR, Willett WC, Buring JE. Alcohol consumption and breast cancer risk in the Women's Health Study. Am J Epidemiol 2007; 165:667-76.

37. Berstad P, Ma H, Bernstein L, Ursin G. Alcohol intake and breast cancer risk among young women. Breast Cancer Res Treat 2008; 108:113-20.

38. Duffy CM, Assaf A, Cyr M, Burkholder G, Coccio E, Rohan $\mathrm{T}$, et al. Alcohol and folate intake and breast cancer risk in the WHI Observational Study. Breast Cancer Res Treat 2009; 116:551-62.

39. Brown LM, Gridley G, Wu AH, Falk RT, Hauptmann $\mathrm{M}$, Colonel LN, et al. Low level alcohol intake, cigarette smoking and risk of breast cancer in AsianAmerican women. Breast Cancer Res Treat 2010; 120:203-10.

40. Terry MB, Knight JA, Zablotska L, Wang Q, John EM, Andrulis IL, et al. Alcohol metabolism, alcohol intake, and breast cancer risk: a sister-set analysis using the Breast Cancer Family Registry. Breast Cancer Res Treat 2007; 106:281-8.

Recebido em 02/Set/2010

Versão final reapresentada em 19/Abr/2011 Aprovado em 06/Mai/2011 